ВОЛОДАРСКИЙ В. А., к.т.н., с.н.с., профессор,

ОРЛЕНКО А. И., к.т.н., доцент, директор (Красноярский институт железнодорожного транспорта)

\title{
О восстановлении ресурса и стратегиях технического содержания устройств
}

Предложены один из возможных способов учета глубины восстановления надежности при проведении ремонтов и аналитические зависимости стоимости ремонта от глубины восстановления ресурса, а также дана классификация стратегий технического содержания устройств.

Ключевые слова: надежность, ресурс, глубина восстановления, ремонт, замена, техническое содержание, стратегия.

\section{Состояние вопроса}

В процессе эксплуатации технических устройств (ТУ) проводится техническое обслуживание, текущие и капитальные ремонты, а также предупредительные замены. Под техническим содержанием будем понимать совокупность мероприятий, направленных на поддержание и возвращение ТУ в работоспособное состояние и восстановления его ресурса.

В настоящее время разработаны методические вопросы только оптимизации замен ТУ [1], когда полностью восстанавливаются их первоначальная надежность. Известно, что при ремонте технических устройств им обычно возвращается лишь часть исходной надежности.

Поэтому как в нашей стране, так и за рубежом уделяется определенное внимание учету глубины восстановления надежности при обслуживании технических устройств. Так в [2] надежность работы системы исследуется при возникновении отказов двух типов. Отказы первого типа устраняются минимальным ремонтом, не изменяющим надежность системы, а отказы второго типа устраняются заменой, полностью обновляющей надежность системы. В [3] при разработке математической модели замены электрооборудования учтена неравноценность отказов, которые разделены на две группы: восстанавливаемые, которые устраняются ремонтом, и невосстанавливаемые, устраняемые заменой. В [4] несовершенство профилактического обслуживания предлагается оценивать вероятностью того, что плановое воздействие приведет систему в состояние аналогичное новому состоянию и вероятностью того, что это воздействие не обновит систему. В [5] также используется вероятность того, что профилактическое обслуживание несовершенно и система при этом отказывает сразу после такого обслуживании.

() В.А. Володарский, А.И. Орленко, 2015
В перечисленных публикациях учитываются только два крайних случая глубины восстановления надежности систем: никакого обновления и полного обновления. Практический же интерес представляют промежуточные значения глубины восстановления надежности между этими крайними случаями.

Естественный процесс старения и износа в [6] учитывается возрастанием числа отказов в каждом последующем межремонтном интервале. В разработанных моделях управления надежностью авиационной техники [7] полнота выполняемых профилактических мероприятий оценивается по изменению интенсивности отказов различных элементов. В работе [8] при проведении профилактического обслуживания введено понятие фактора улучшения, который предложено оценивать экспертным путем. Вопросы учета глубины восстановления надежности рассматривались в перечисленных публикациях только на теоретическом уровне и далеки от практической реализации.

Относительно в лучшей степени вопросы оценки глубины восстановления надежности решаются при ремонте машин в сельском хозяйстве, где введено понятие степени восстановления ресурса при капитальном ремонте [9]. Однако при расчете оптимальных сроков ремонта машин в [10] использованы известные в теории надежности модели оптимизации [11], которые построены в предположении полного восстановления надежности при заменах, как в предупредительном порядке, так и в случае отказа устройств. Это, как известно, является большой идеализацией реального процесса обновления при ремонте устройств.

Цель статьи - предложить один из возможных способов учета глубины восстановления надежности при проведении ремонтов и аналитические зависимости стоимости ремонта от глубины восстановления ресурса, а также дать классификацию стратегий технического содержания устройств. 
Учет глубины восстановления ресурса

В процессе эксплуатации ТУ неизбежны его отказы по различным причинам, которые можно классифицировать в зависимости от сложности их устранения. Важное технико-экономическое значение при этом имеет выбор места и метода устранения отказа. По этому признаку целесообразно все отказы разделить на две группы: отказы работоспособности и ресурсные отказы. Для устранения отказов первой группы не требуются трудоемкие работы, а работоспособность ТУ восстанавливаются заменой, ремонтом или регулировкой неисправных элементов в объеме технического обслуживания (ТО) или непланового текущего ремонта (ТР). При этом остаточный ресурс ТУ остается прежним. Такой способ устранения отказов прост, не требует высокой квалификации обслуживающего персонала и сложного оборудования. Его можно использовать непосредственно на месте эксплуатации. Преимущество этого способа - возможность полного использования ресурса большинства элементов ТУ.

Под ресурсным отказом будем понимать такое событие, которое характеризуется либо физическим выходом ТУ из строя как целого, либо такими отказами его элементов, при которых целесообразно восстановление до определенного уровня ресурса всего ТУ. Для устранения такого отказа необходимо выполнить трудоемкие разборные, ремонтные, сборные, регулировочные, обкаточные и другие работы. Такой способ устранения отказа более сложен, и для его проведения требуются специальные производственные условия ремонта. Преимущество этого способа: восстановление установленного нормативно-технической документацией ресурса и повышение, таким образом, надежности ТУ. Ресурсные отказы устраняются проведением непланового капитального ремонта (КР) или неплановой заменой. В первом случае происходит частичное, а во втором случае - полное восстановление ресурса ТУ.

Все ремонтно-обслуживающие воздействия в процессе эксплуатации ТУ можно классифицировать по трем признакам: по моменту проведения работы плановая (заранее известен календарный момент времени) или неплановая (календарный момент проведения не известен); по характеру работы предупредительная или аварийная; по глубине восстановления ресурса - никакого обновления не производится (выполняется ТО или ТР); частичное восстановление (выполняется КР) и полное восстановление (выполняется замена).

Для учета глубины восстановления ресурса при предупредительном и аварийном ремонте ТУ предлагается использовать два параметра $[12,13]$ :

$q$ - вероятность ресурсных отказов, которые требуют для их устранения проведения капитального ремонта или замены ТУ. Используя статистику об отказах, параметр $q$ определяется как $q=m_{1} / m$, где $m_{1}$ - количество ресурсных отказов; $m$ - общее количество отказов;

$a$ - «возраст» ТУ после проведения капитального ремонта.

Изменение интенсивности ресурсных отказов при проведении капитальных ремонтов и замены ТУ представлено на рис. 1 , где $T_{\text {дp }}, T_{\mathrm{mp}}$ и $T_{\mathrm{p}}-$ соответственно, доремонтный, межремонтный и полный ресурс ТУ. Полным обновлением назовем такое, при котором ТУ как бы «возвращается» в состояние, которое оно имело в момент начала эксплуатации (точка 0), когда интенсивность ресурсных отказов равна нулю. Это соответствует тому, что проведена предупредительная замена.

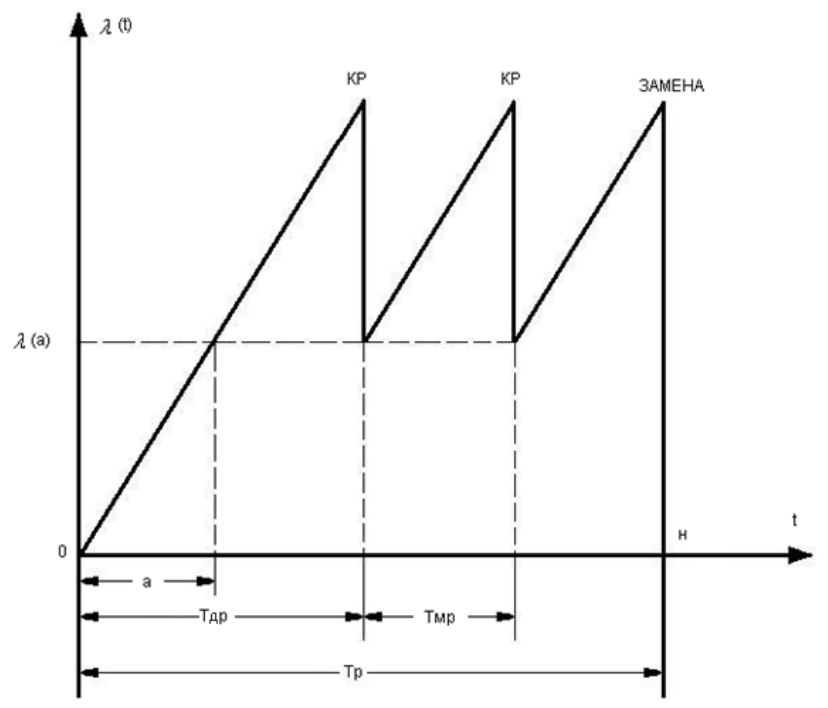

Рис. 1. Изменение интенсивности ресурсных отказов при проведении капитальных ремонтов и замены

При проведении ремонта параметр а, предлагается оценивать как $\mathrm{a}=T_{\text {дp }}-T_{\mathrm{mp}} \quad$ (см. рис. 1). В дальнейшем при разработке математических моделей оптимизации предупредительных замен и ремонтов для оценки глубины восстановления ресурса целесообразно использовать безразмерную величину,

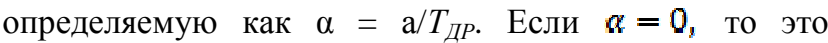
означает, что проведена замена ТУ. Если проводится КР, например, через время $\tau$ то «возраст» ТУ уменьшается от значения $\tau$ до $\alpha \tau$.

Аналитические зависимости стоимости ремонта от глубины восстановления ресурса могут быть получены исходя из следующего. При полном восстановлении pecypca, когда проводится замена ТУ и 
$\alpha=0$, отношение стоимости капитального ремонта $\gamma_{1}$ к стоимости нового ТУ $\gamma$ равно единице (см. рис. 2, точка 1,0 на оси ординат). Это означает, что $T_{\mathrm{mp}}=T_{\text {др }}$. При $\gamma_{1}=0$, когда КР не проводится и $T_{\text {мр }}=0$, глубина восстановления ресурса равна единице (см. рис. 2, точка 1,0 на оси абсцисс). Исходя из гипотезы, что стоимость капитального ремонта прямо пропорциональна глубине восстановления ресурса ТУ, можно получить линейную зависимость вида $\gamma_{1} / \gamma=1-\alpha$, представленную на рис. 2 прямой линией 1. Естественно предположить, что для более нового ТУ кривые таких зависимостей будут проходить ниже прямой 1 , а для более старого - выше прямой 1. Для аппроксимации таких зависимостей предлагается использовать степенную функцию вида

$\gamma_{1} / \gamma=1-\alpha^{\mathrm{C}}$

где $c$ - параметр формы, принимает различные положительные значения.

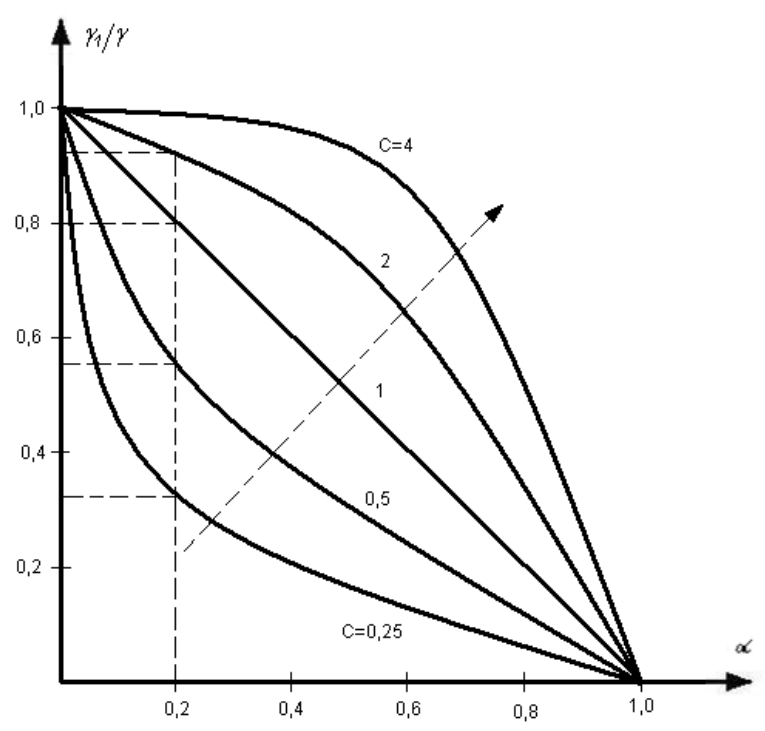

Рис. 2. Кривые зависимостей стоимости капитального ремонта от глубины восстановления ресурса

На рис. 2 в качестве примера представлен набор кривых при параметре $c$ равным 0,$25 ; 0,5 ; 2,0$ и 4,0. С увеличением срока службы ТУ параметр формы возрастает, и зависимости стоимости от глубины восстановления ресурса при ремонте смещаются, как показано на рис. 2 стрелкой. Это означает, что при одной и той же глубине восстановления ресурса, например, $\alpha=0,2$, для более нового ТУ стоимость капитального ремонта будет меньше (см. пунктирные линии на рис. 2).
Из (1) могут быть получены зависимости стоимости капитального ремонта как функция глубины восстановления ресурса и стоимости нового оборудования вида

$\gamma_{1}(\alpha)=\gamma\left(1-\alpha^{c}\right)$

Зависимости (2) могут быть использованы при разработке математических моделей оптимизации периодичности предупредительных замен и ремонтов ТУ. Для уточнения и использования в том или ином случае конкретных из предложенных аналитических зависимостей можно использовать метод экспертных оценок.

При разработке математических моделей оптимизации предупредительных замен и ремонтов ТУ необходимо учитывать, что интенсивность отказов изменяется в общем случае, как показано на рис. 3, где $x$ - время в единицах ресурса. Отказы работоспособности, вероятность которых $\bar{q}=1-q$, устраняются в порядке технического обслуживания или непланового текущего ремонта. Эту операцию в терминах теории надежности [11] назовем минимальным аварийным ремонтом (МАР). При проведении МАР интенсивность отказов не изменяется (см. рис. 3). Ресурсные отказы с вероятностью $q$ устраняются аварийным капитальным ремонтом (АР). $\mathrm{C}$ оптимальной периодичностью $x_{0}$ проводятся предупредительные капитальные ремонты (ПР) с глубиной восстановления ресурса $\alpha$. После проведении АР и ПР интенсивность отказов снижается до $\lambda(\alpha)$ (см. рис. 3). С периодичностью $x_{\mathrm{p}}$ проводятся предупредительные замены (ПЗ), снижающие интенсивность отказов ТУ до нуля. Оптимальное значение интенсивности отказов в период проведения ПР и ПЗ составляет $\lambda\left(x_{0}, \alpha\right)$ (см. рис. 3$)$.

Таким образом, при оптимизации предупредительных замен и ремонтов имеются два управляемых параметра: периодичность $x$ и глубина восстановления ресурса $\alpha$. При этом показатели надежности работы ТУ, необходимые для разработки моделей оптимизации предупредительных замен и ремонтов, определяются следующим образом. При проведении предупредительного ремонта с периодичностью $x$ и глубиной $\alpha$, вероятность безотказной работы определяется как

$P(x / \alpha)=P(x+\alpha) / P(\alpha)$,

а вероятность безотказной работы по ресурсным отказам определяется из выражения

$G(x / \alpha)=[P(x / \alpha)]^{q}=[P(x+\alpha) / P(\alpha)]^{q}$. 


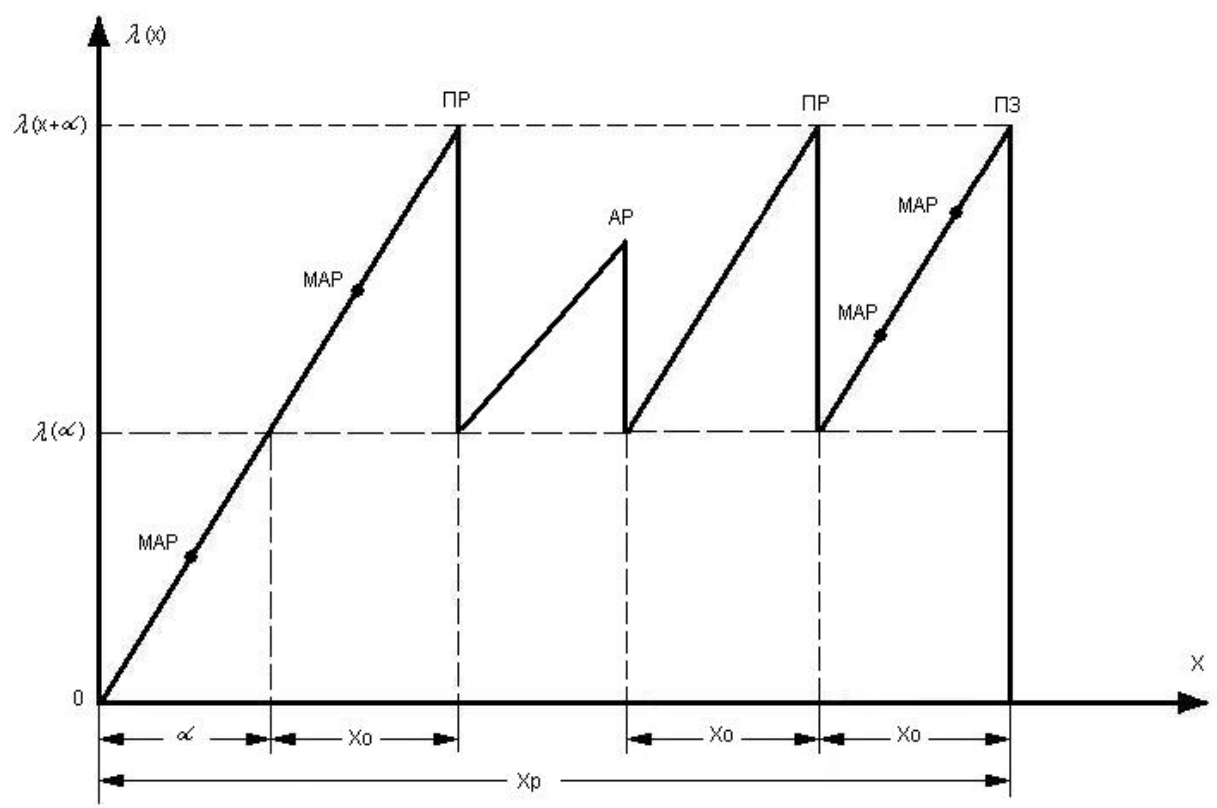

Рис. 3. Изменение интенсивности отказов при проведении аварийных и предупредительных ремонтов и замен

При проведении предупредительных замен, как частный случай из выражения (3) при $\alpha=0$, получим $G(x)=[P(x)]^{a}$. Это выражение эквивалентно выражению, полученному в [2].

Интенсивность отказов при проведении предупредительного ремонта ТУ с периодичностью $x$ и глубиной восстановления ресурса $\alpha$ определяется из выражения

$\lambda(x / \alpha)=-P^{\prime}(x / \alpha) / P(x / \alpha)=\lambda(x+\alpha)$,

а интенсивность ресурсных отказов -

$\lambda_{\mathrm{p}}(x / \alpha)=-G^{/}(x / \alpha) / \mathrm{G}(x / \alpha)=q \lambda(x+\alpha)$.

Тогда интенсивность отказов работоспособности ТУ равна

$\lambda_{\mathrm{r}}(x / \alpha)=\lambda(x / \alpha)-\lambda_{\mathrm{p}}(x / \alpha)=\bar{q} \lambda(x+\alpha)$.

При $\alpha=0$ получим:

$\lambda_{\mathrm{p}}(x)=q \underline{\lambda}(x) ; \quad \lambda_{\mathrm{s}}(x)=\bar{q} \lambda(x)$.

\begin{tabular}{|c|c|c|}
\hline $\begin{array}{l}\text { Классификация } \\
\text { содержания }\end{array}$ & стратегий & технического \\
\hline
\end{tabular}

В зависимости от наличия тех или иных предупредительных и аварийных ремонтов и замен, то есть в зависимости от значений, предложенных для учета глубины восстановления ресурса параметров, целесообразно выделить следующие стратегии технического содержания устройств:

$\alpha>0 ; q=1$ - предупредительный капитальный ремонт по наработке, когда выполняются ПР и АР;

$\alpha>0 ; q=0$ - предупредительный капитальный ремонт с минимальным аварийным ремонтом, когда выполняются ПР и МАР;

$\alpha>0 ; 0<q<1$ - предупредительный капитальный ремонт по наработке с полным или минимальным аварийным ремонтом, когда выполняются ПР, АР или MAP;

$\alpha=0 ; q=1$ - предупредительная замена по наработке, когда выполняются ПЗ и аварийная замена (A3);

$\alpha=0 ; q=0$ - предупредительная замена с минимальным аварийным ремонтом, когда выполняются ПЗ и МАР;

$\alpha=0 ; 0<q<1$ - предупредительная замена по наработке с аварийной заменой или минимальным аварийным ремонтом, когда выполняются ПЗ, АЗ или MAP;

$\alpha \geq 0 ; q=0$ - предупредительный капитальный ремонт и замена с минимальным аварийным ремонтом, когда выполняются ПЗ, ПР и МАР;

$\alpha \geq 0 ; q=1$ - предупредительный капитальный ремонт и замена с полным аварийным ремонтом, когда выполняются ПЗ, ПР и АР;

$\alpha \geq 0 ; \quad 0<q<1$ - предупредительный капитальный ремонт и замена с полным или минимальным 
аварийным ремонтом, когда выполняются ПЗ, ПР, АР или МАР.

\begin{tabular}{l}
\hline Выводы \\
\hline 1. Для учета глубины восстановления ресурса \\
при аварийном и предупредительном ремонте \\
целесообразно использовать два параметра: \\
вероятность ресурсных отказов, требующих \\
проведения капитального ремонта или замены ТУ; \\
«возраст», определяемый как разница между \\
доремонтным и межремонтным ресурсом ТУ. Эти \\
параметры адекватно отражают реальные процессы \\
обновления после любого вида ремонта. При этом \\
можно определить распределения показателей \\
надежности без изменения их функциональной \\
структуры, которые необходимы для разработки \\
математических моделей оптимизации периодичности \\
предупредительных ремонтов и замен технических \\
устройств.
\end{tabular}

2. При разработке моделей оптимизации предупредительных ремонтов рекомендуется использовать предложенные аналитические зависимости стоимости ремонта от глубины восстановления ресурса технических устройств.

3. Стратегии технического содержания ТУ рекомендуется классифицировать в зависимости от значений параметров, предложенных для учета глубины восстановления ресурса технических устройств.

1. Методика оптимизации периодичности проведения замен технических устройств.- М.: Издательство стандартов, 1975. - 21 с.

2. Beichelt T., Fisher K. General failure model applied to preventive maintenance policies//IEEE Transactions on reliability.- 1980. - Vol. R-29, N 1.- P. 39-41.

3. Разгильдеев Г. И., Захарова А.Г. Математическая модель замены электрооборудования с учетом неравноценности отказов // Известия вузов. Энергетика.- 1981. - №9. - С. 79-84.

4. Helvik B. Periodic maintenance on the effect of imperfectness $/ / 10^{\text {th }}$ Int. Symp. Fault Tolerant Comput. - Kyoto, Oct. 1-3, 1980. - P. 204-206.

5. Murthy D.N.P., Ngugen D.G. Optimal age - policy with imperfect preventive maintenance// IEEE Transactions on reliability. - 1981. - Vol. R-30, N 1.P. 80-81.

6. Рудь Ю.С. Оптимизация технического обслуживания технологического оборудования горно-обогатительных комбинатов // Известия вузов. Горный журнал - 1980. - № 12 - С . 47-51.

7. Моломин В.П. Управление надежностью авиационной техники. - М.: Машиностроение, 1981.- 200c.
8. Malik M. A. K. Reliable preventive maintenance scheduling // AIIE Trans. - 1979.- Vol. 11.- P. 221228.

9. Черепанов С.С. Техническое обслуживание и ремонт машин в сельском хозяйстве.- М.: Колос, 1979.- 288c.

10. Хальфин М. А. Управление надежностью машин в эксплуатации // Механизации электрификации сельского хозяйства. - 1982- № 1 - С. 46-52.

11. Барлоу Р., Прошан Ф. Математическая теория надежности. - М.: Советское радио, 1969 - 488c.

12. Володарский В.А. К вопросу оптимизации периодичности предупредительных замен и ремонтов электрооборудования // Промышленная энергетика. - 1988. - № 8. - С. 34 -36.

13. Володарский В.А., Орленко А.И. О надежности подвижного состава, прошедшего ремонт // Надежность. - 2015. - № 1.- С. 25-28.

Volodarsky V.A., Orlenko A.I. On the recovery of the resources and the strategies of technical content of devices. One of the possible ways of accounting the depth of the recovery of reliability while repairing and analytical dependences of repair cost upon the depth of resource recovery have been proposed. The classification of the strategies of device technical content has been given. To account for the depth of resource recovery in preventive and emergency repair it is proposed to use two parameters: the probability of resource failures, which require for their removal overhaul or replacement devices, and "age" devices after overhaul as the difference between a repair and overhaul resource. Provides analytical expressions for the cost of repairs from the depth of resource recovery that can be used in the development of mathematical models of optimization of the periodicity of preventive replacements and repair devices.

Formulae for determination of reliability parameters, depending on the frequency of repairs and depth recovery of a resource that can be used in the development of mathematical models of optimization of the periodicity of preventive replacements and repair devices. Depending on the values proposed to account for the depth recovery resource settings presents a detailed classification of the strategies of technical content devices.

Key words: reliability, resource, recovery depth, repair, replacement, maintenance, strategy.

Рецензент д.т.н., профессор Терсков В.А. (Красноярский институт железнодорожного транспорта)

Поступила 12.05.20152.

Volodarsky Vladislav A., PhD, senior researcher, associate professor «Krasnoyarsk Institute of Railway Transport», Krasnoyarsk, Russia.

Orlenko Aleksey I., PhD, associate professor «Krasnoyarsk Institute of Railway Transport», Krasnoyarsk, Russia. 